\title{
Prevalence of Metabolic Syndrome in Treatment Naïve Rheumatoid Arthritis and Correlation With Disease Parameters
}

\author{
Purneshwar Kumar PANDEY, Abhijit SWAMI, Tanuj Kumar BISWAS, Riturag THAKURIA \\ Silchar Medical College and Hospital, Department of Medicine, Silchar, India
}

\begin{abstract}
Objectives: This study aims to assess the prevalence of metabolic syndrome (MetS) in treatment naïve rheumatoid arthritis (RA) in an Indian population and correlate RA disease characteristics with presence of MetS.

Patients and methods: The study included 84 RA patients (18 males, 66 females; mean age $44.8 \pm 12.5$ years; range 18 to 72 years) diagnosed according to 2010 American College of Rheumatology-European League Against Rheumatism classification criteria who were treatment naïve or did not receive disease modifying antirheumatic drugs for more than six weeks and 120 age and sex-matched apparently healthy controls ( 35 males, 85 females; mean age $44.1 \pm 12.7$ years; range 18 to 75 years). The frequency of MetS was assessed using National Cholesterol Education ProgramAdult Treatment Panel III 2004 revised criteria. Patients were also assessed in terms of disease activity, using disease activity score 28 erythrocyte sedimentation rate. Logistic regression was used to identify predictors of MetS in RA.

Results: Metabolic syndrome was found in 39.28\% of RA group and 20\% of control group according to National Cholesterol Education ProgramAdult Treatment Panel III 2004 ( $p<0.005)$. MetS was most commonly detected in the 51 to 60 age group (65\%). RA group was significantly more likely to have low high-density lipoprotein (63.09\%), high triglyceride (53.57\%), elevated blood pressure (41.66\%) levels, and elevated waist circumference (38.09\%). In RA group, disease activity score 28 (odds ratio: 6.51, confidence interval: 1.19-35.46 p=0.03), C-reactive protein (odds ratio: 1.13 , confidence interval: $1.05-1.21 \mathrm{p}<0.001$ ), and duration of disease (odds ratio: 1.82 , confidence interval: $1.04-3.18 \mathrm{p}=0.03$ ) remained independent predictors for presence of MetS in RA.

Conclusion: The frequency of MetS was higher in RA group compared to control group. Higher systemic inflammatory marker, disease duration, and disease activity score 28 remained independent predictors associated with presence of MetS. These findings suggest that RA patients should be screened early for presence of MetS to check for and reduce risk of atherosclerotic vascular diseases.

Keywords: Disease activity; metabolic syndrome; rheumatoid arthritis.
\end{abstract}

Rheumatoid arthritis (RA) is a chronic autoimmune inflammatory disease of unknown etiology that is relatively common and disabling, characterized by progressive joint disorder, significant pain, and functional disability, which may cause premature mortality and compromised quality of life. ${ }^{1}$

Rheumatoid arthritis is associated with an increased risk of atherosclerotic vascular disease that accounts for about half of all deaths in this population (standardized mortality ratio of 1.5 ) including myocardial infarction, cerebrovascular accidents, and heart failure. ${ }^{2}$ Several cardiovascular risk factors are present at higher frequency in patients with RA. In addition, chronic inflammation and drugs used in treatment strategies and sedentary lifestyle may play an important role in increasing the risk of cardiovascular disease in RA patients. ${ }^{3,4}$ Epidemiological data suggest that RA is an independent risk factor for atherosclerotic vascular diseases though increased rates have not been explained by traditional risk factors, use of steroids or non-steroidal anti-inflammatory drugs, or shared genetic features. ${ }^{5}$

The development of accelerated atherosclerosis and increased risk of cardiovascular disease in patients with RA may be influenced by occurrence

Received: February 04, 2016 Accepted: June 26, 2016 Published online: October 13, 2016

Correspondence: Purneshwar Kumar Pandey, MD. Silchar Medical College and Hospital, Department of Medicine, 788014 Silchar, India.

Tel: 08471955150 e-mail: purneshwar2kumar@gmail.com

(2017 Turkish League Against Rheumatism. All rights reserved. 
of metabolic syndrome (MetS). ${ }^{6}$ MetS describes a constellation of major risk factors for cardiovascular diseases such as atherogenic dyslipidemia, obesity, hypertension, and diabetes. It is measured in terms of waist circumference, triglyceride levels, fasting blood glucose, and high-density lipoprotein (HDL) level. Its underlying pathophysiology is thought to be insulin resistance. ${ }^{7}$

There are five definitions for metabolic syndrome by National Cholesterol Education Program-Adult Treatment Panel (NCEP-ATP) III 2001, NCEP-ATP 2004, World Health Organization, International Diabetes Federation, and European group for study of insulin resistance. However, the relative value of the different MetS definitions in terms of prognosis and management has been established to be similar. Nevertheless, the NCEP-ATP III and International Diabetes Federation definitions are most widely used. ${ }^{8}$

There is paucity of data regarding the prevalence of MetS in RA patients in Indian population. Asian Indians are a metabolically disadvantaged ethnic group with high prevalence of obesity, diabetes, and dyslipidemia. ${ }^{9}$ Thus, in this study, we aimed to assess the prevalence of MetS in treatment naïve RA in an Indian population and correlate RA disease characteristics with presence of MetS.

\section{PATIENTS AND METHODS}

This cross-sectional study included 84 patients with RA (18 males, 66 females; mean age $44.8 \pm 12.5$ years; range 18 to 72 years) attending Silchar Medical college and Hospital, Srimanta Sankaradeva University of Health Sciences situated in eastern India between January 2014 and June 2015 and fulfilling 2010 American College of Rheumatology/European League against Rheumatism classification criteria for RA. ${ }^{10}$

In this study, patients with RA were evaluated for prevalence of metabolic syndrome. The study group consisted of RA patients who were treatment naïve or did not receive disease modifying antirheumatic drugs (DMARDs) for more than six weeks before attending hospital due to financial constraints or access to healthcare facilities.
Patients with inflammatory diseases other than RA such as neoplastic disorders, chronic kidney disease, chronic liver disease, or with chronic infections like human immunodeficiency virus, or diseases known to cause dyslipidemia other than human immunodeficiency virus, e.g. hypothyroidism, nephrotic syndrome, or who received lipid or urate lowering drugs, irregular treatment with DMARDs, hormone replacement therapy, or patients aged $<16$ years, female patients on oral contraceptive pills, pregnant patients, patients with a diagnosis of familial dyslipidemia, or patients who received DMARD for longer than six weeks were excluded.

One hundred and twenty (35 males, 85 females; mean age $44.1 \pm 12.7$ years; range 18 to 75 years) apparently healthy volunteers from general population of Cachar served as a control group.

A written informed consent was obtained from all the participant. The study was approved by Research and Ethical Review Board of Silchar Medical College and Hospital. The study was conducted in accordance with the principles of the Declaration of Helsinki.

Demographic features, disease specific variables (disease duration, duration of morning stiffness, tender and swollen joint count), risk factors for MetS such as age, lifestyle, smoking, alcohol, physical activity etc., drug use, menstrual history, comorbid conditions, and family history of rheumatic and cardiovascular diseases were documented for each patient.

Patients were also evaluated in terms of disease activity, using disease activity score 28 (DAS28) erythrocyte sedimentation rate (ESR). Pain and general health were measured by visual analog scale.

Height, weight, waist circumference, and hip circumference were noted for both patient and control groups. Waist circumference was measured with a non-stretchable tape, placed directly on skin, at end of expiration at midway between lower costal margin and upper border of iliac crest. Body mass index (BMI) was calculated as body weight divided by square of height $\left(\mathrm{kg} / \mathrm{m}^{2}\right)$. In accordance with World Health Organization standard for Asian population, BMI $<18.5 \mathrm{~kg} / \mathrm{m}^{2}$ was considered underweight, 
Table 1. Demographic and anthropometric parameters of RA patients and controls

\begin{tabular}{|c|c|c|c|c|c|}
\hline & Contrc & roup $(n=120)$ & RA & up $(n=84)$ & \\
\hline & $\%$ & Mean $\pm S D$ & $\%$ & Mean \pm SD & $p$ \\
\hline Age (years) & & $44.1 \pm 12.8$ & & $44.8 \pm 12.7$ & 0.6962 \\
\hline Female & 70.83 & & 78.57 & & 0.2571 \\
\hline Weight $(\mathrm{kg})$ & & $59.3 \pm 8.5$ & & $62 \pm 10.1$ & 0.0397 \\
\hline Height $(\mathrm{cm})$ & & $159.5 \pm 6.1$ & & $159.6 \pm 6.6$ & 0.9469 \\
\hline Body mass index $\left(\mathrm{kg} / \mathrm{m}^{2}\right)$ & & $23.2 \pm 2.5$ & & $24.2 \pm 2.5$ & 0.0039 \\
\hline Waist circumference $(\mathrm{cm})$ & & $83.3 \pm 6.9$ & & $86.4 \pm 7.9$ & 0.0032 \\
\hline Waist hip ratio & & $0.9 \pm 0.1$ & & $0.9 \pm 0.1$ & 0.2352 \\
\hline Systolic blood pressure $(\mathrm{mmHg})$ & & $125 \pm 20$ & & $135 \pm 21.1$ & 0.0006 \\
\hline Diastolic blood pressure $(\mathrm{mmHg})$ & & $79 \pm 11$ & & $82.1 \pm 10.7$ & 0.046 \\
\hline
\end{tabular}

18.5-22.9 as normal, 23-27.49 as overweight, and $>27.5 \mathrm{~kg} / \mathrm{m}^{2}$ as obese. Blood pressure was measured using mercury sphygmomanometer in sitting position after five minutes of rest.

Biochemical tests were performed from venous sample after an overnight fast of 10 hours. Fasting blood glucose, serum creatinine, triglyceride, HDL cholesterol, and total cholesterol were measured using autoanalyzer (Beckman Coulter AU480 ${ }^{\mathrm{TM}}$ system). Very low-density lipoprotein and lowdensity lipoprotein (LDL) cholesterols were estimated using indirect methods [serum very $\mathrm{LDL}=$ serum triglycerides/5], [serum $\mathrm{LDL}=$ total cholesterol HDL cholesterol - very LDL]. Immunoglobulin $\mathrm{M}$, rheumatoid factor, and anti-cyclic citrullinated peptide were assessed using enzyme-linked immunosorbent assay. ESR was measured using Wintrobe's method and C-reactive protein (CRP) was measured using turbidimetric immunoassay with semiautomatic analyzer (ERBA Chem 5x).

\section{Statistical analysis}

The collected data was compiled, tabulated and analyzed in terms of descriptive statistics using IBM SPSS version 22.0 software (IBM
Corporation, Armonk, NY, USA). Continuous variables were presented as mean \pm standard deviation and categorical variables were expressed as frequencies and percentages. Nominal categorical data between the groups were compared using Chi-squared test or Fisher's exact test as appropriate. $\mathrm{P}<0.05$ was considered statistically significant. Graphical and diagrammatic representations were presented whenever felt necessary. Multivariate logistic regression models were constructed and odds ratio and 95\% confidence interval were calculated to investigate the independent predictors of individual RA-related characteristics and MetS in patients with RA.

\section{RESULTS}

Baseline characteristics of both patient and control groups are shown in Table 1. Smoking, alcohol, and postmenopausal statuses in females were similar in both groups. Mean waist circumference, BMI, and systolic blood pressure were significantly higher in RA group than control group $(p<0.005)$.

Table 2. Biochemical parameters-rheumatoid arthritis and controls

\begin{tabular}{|c|c|c|c|c|c|}
\hline & \multicolumn{2}{|c|}{ Control group $(n=120)$} & \multicolumn{2}{|c|}{ RA group ( $\mathrm{n}=84$ ) } & \multirow[b]{2}{*}{$\mathrm{p}$} \\
\hline & Median & IQR & Median & IQR & \\
\hline Total cholesterol (mg/dL) & 152 & $125.15-179$ & 149 & $128.70-175.02$ & 0.3330 \\
\hline Triglycerides (mg/dL) & 121 & 93-192 & 154 & 130.0-192.5 & 0.0419 \\
\hline $\mathrm{HDL}(\mathrm{mg} / \mathrm{dL})$ & 40.32 & $32.47-45.3$ & 32 & $23.87-45$ & 0.0001 \\
\hline $\mathrm{LDL}(\mathrm{mg} / \mathrm{dL})$ & 82 & $68-106.25$ & 80 & $60-110$ & 0.750 \\
\hline Uric acid & 4.79 & $3.67-6.10$ & 4.41 & $3.43-5.49$ & 0.2049 \\
\hline FBG (mg/dL) & 83.5 & $75-96.25$ & 98.5 & $87.75-118$ & 0.001 \\
\hline
\end{tabular}


Table 3. Prevalence of components of metabolic syndrome-rheumatoid arthritis and controls

\begin{tabular}{lccccccr}
\hline & \multicolumn{2}{c}{ Control group $(\mathrm{n}=120)$} & & \multicolumn{2}{c}{ RA group $(\mathrm{n}=84)$} \\
\cline { 2 - 3 } & Frequency & $\%$ & & Frequency & $\%$ & \\
\hline Elevated waist circumference $(\mathrm{cm})$ & 20 & 16.66 & & 32 & 38.09 & 0.0010 \\
Elevated triglycerides $(\mathrm{mg} / \mathrm{dL})$ & 38 & 31.66 & & 45 & 53.57 & 0.0023 \\
Reduced HDL (mg/dL) & 57 & 47.5 & & 53 & 63.09 & 0.0326 \\
Elevated BP (mmHg) & 30 & 25 & & 35 & 41.66 & 0.0146 \\
Elevated fasting blood glucose (mg/dL) & 15 & 12.5 & & 23 & 27.38 & 0.0101 \\
\hline HDL: High-density lipoprotein; RA: Rheumatoid arthritis; BP: Blood pressure. & & & & \\
\hline
\end{tabular}

Biochemical parameters are demonstrated in Table 2. Median HDL level was significantly lower in RA group than in control group. Similarly, median triglyceride and fasting blood glucose levels were significantly higher in RA group than in control group. However, no difference was noted between both groups with respect to total cholesterol, serum uric acid, and LDL cholesterol levels.

The criteria for MetS are presented in Table 3. The most common abnormality noted in RA was reduced HDL cholesterol (63.09\%) followed by increased triglyceride (53.57\%) levels. However, the most significant difference between RA and control groups were elevated waist circumference and elevated triglyceride level.

Characteristics of RA patients are summarized in Table 4. The mean duration of disease was $5.1 \pm 2.5$ years. Of RA patients, $78.57 \%$ were rheumatoid factor positive and $84.52 \%$ were anti-cyclic citrullinated peptide positive. Mean CRP and ESR were $52.39 \pm 23.39 \mathrm{mg} / \mathrm{dL}$ and $43.3 \pm 24.82 \mathrm{~mm}$ at end of first hour, respectively. The mean DAS28 score was $4.8 \pm 0.84$ and proportions of patients with mild $(<3.2)$, moderate (3.2-5.1), and severe (>5.1) disease were 5.95\%, $54.76 \%$, and $39.28 \%$, respectively.

Table 4. Rheumatoid arthritis disease parameters

\begin{tabular}{lcc} 
Parameters & $\%$ & Mean \pm SD \\
\hline CRP (mg/L) & $58.0 \pm 24.9$ \\
ESR (mm at end of first hour) & $48.1 \pm 25.6$ \\
DAS28 score & $4.8 \pm 0.8$ \\
Duration of disease (years) & $5.1 \pm 2.5$ \\
Rheumatoid positive & \\
Anti-CCP positive & 78.57 & \\
\hline SD: Standard deviation; CRP: C-reactive protein; ESR: Erythrocyte \\
sedimentation rate; DAS28: Disease activity score 28; Anti-CCP: Anti- \\
cyclic citrullinated peptide.
\end{tabular}

The prevalence of MetS in RA group was $39.28 \%$, while it was $20 \%$ in the control group $(p<0.005)$. In univariate analysis, females with RA having MetS were older, had less regular intentional exercise, longer disease duration, higher CRP, higher ESR, and higher DAS28 score $(p<0.001)$ (Table 5). In multivariate analysis, there was a significant association with duration of disease (odds ratio: 1.82, confidence interval: 1.04-3.18 $\mathrm{p}=0.03$ ), DAS 28 (odds ratio: 6.51, confidence interval: 1.19-35.46 $\mathrm{p}=0.03$ ), and CRP (odds ratio: 1.136, confidence interval: $1.05-1.21 \mathrm{p}<0.001$ ) \{adjusted for age and physical activity\} with prevalence of MetS in RA (Table 6).

\section{DISCUSSION}

This study was conducted in eastern India in patients with RA and detected that prevalence of MetS was significantly higher in RA group compared to control group. Among RA patients with metabolic syndrome, more patients were in the age group of 51 to 60 years with a female preponderance.

Most studies on prevalence of MetS in patients with RA have included patients with DMARD therapy but only a few have reported on treatment naïve patients. Apart from that, a limited number of studies have been reported from the Indian subcontinent.

The frequency of MetS has varied markedly between different studies, ${ }^{11}$ most likely because of the lack of accepted criteria for the definition of MetS. The frequency of MetS (40\%) shown by Karvounaris et al. ${ }^{12}$ (defined according to the NCEP-ATP III criteria) in RA patients was comparable with their control population. In South Asia, Dodani et al. ${ }^{13}$ found that the frequencies of MetS in patient in rheumatoid arthritis were 


\begin{tabular}{|c|c|c|c|}
\hline & MetS present & MetS absent & \\
\hline & Mean \pm SD & Mean \pm SD & $p$ \\
\hline Age (years) & $51.8 \pm 7.9$ & $40.2 \pm 13.1$ & $<0.001$ \\
\hline Body mass index $\left(\mathrm{kg} / \mathrm{m}^{2}\right)$ & $26.0 \pm 2.2$ & $23.0 \pm 1.8$ & $<0.001$ \\
\hline Disease duration (years) & $6.6 \pm 1.6$ & $4.0 \pm 2.5$ & $<0.001$ \\
\hline Erythrocyte sedimentation rate ( $\mathrm{mm}$ at end of first hour) & $44.8 \pm 27.7$ & $34.4 \pm 18.1$ & 0.03 \\
\hline C-reactive protein (mg/dL) & $74.8 \pm 17.2$ & $38.8 \pm 12.5$ & $<0.001$ \\
\hline Disease activity score 28 & $5.4 \pm 0.6$ & $4.4 \pm 0.7$ & $<0.001$ \\
\hline
\end{tabular}

$13.3 \%$ and $40 \%$, respectively, according to World Health Organization and NCEP-ATP III criteria.

In the literature, prevalence of MetS varied considerably using the NCEP 2001; the prevalence ranged from $17 \%$ in Mexican, ${ }^{14}$ 19\% in South African, ${ }^{15} 19.9 \%$ in Dutch, ${ }^{16} 38.3 \%$ in English, ${ }^{17}$ to $41.5 \%$ in Swedish, ${ }^{18} 42 \%$ in American, ${ }^{19}$ and $44 \%$ in Greek ${ }^{12}$ patients with RA. Such diversity can be explained by differences in the baseline and disease characteristics. ${ }^{12-19}$ Furthermore, in an Indian study, Aman et al. ${ }^{20}$ also reported a higher prevalence of MetS in RA patients than general population (31.57\% vs $14.9 \%$, respectively).

Chronic inflammation seen in patients with RA is one of the important factors which links it to both MetS and atherosclerosis. ${ }^{21}$ Proinflammatory cytokines, tumor necrosis factor-alpha, and interleukin- 6 in patients with RA contribute to insulin resistance. Insulin resistance leads to other metabolic disturbances like hyperglycemia and dyslipidemia which independently contribute to atherosclerosis and cardiovascular risk. Proinflammatory cytokines are also independently involved in pathogenesis of atherosclerosis through production of acute phase reactant $\mathrm{C}$-reactive protein. Thus multiple mechanisms including inflammation, insulin resistance, or dyslipidemia increase burden of cardiovascular risk in RA patients. $^{22}$

Our study found lower HDL cholesterol, higher triglyceride, and higher fasting blood glucose levels in RA group as compared to control group. Similar findings were reported by Dessein et al. ${ }^{15}$ in their study on RA patients and controls. The lipid profile of RA patients has been evaluated in several studies that have reported dyslipidemic ${ }^{23,24}$ as well as normal pattern ${ }^{25}$ in RA patients when compared to controls. The discrepancies observed in the lipid values in various studies could be due to differences in the population studied as well as disease activity.

Our study also reported higher waist circumference, weight, and BMI in RA group than in control group along with limited physical activity. These findings are in concordance with the previous studies. ${ }^{26}$

We also found significantly higher mean blood pressure in RA group than control group and higher prevalence of hypertension in RA group. Dao et al. ${ }^{27}$ reported a similar higher prevalence of hypertension in RA patients. Moreover, Karvounaris et al. ${ }^{12}$ showed a significant correlation between systolic blood pressure and DAS28 score which was quite high in our study.

Table 6. Odds ratios for having the metabolic syndrome in patients with rheumatoid arthritis

\begin{tabular}{lcc}
\hline & Odds ratios $(95 \% \mathrm{CI})$ & $p$ \\
\hline Disease duration & $1.825(1.046$ to 3.186$)$ & 0.034 \\
Rheumatoid factor & $1.092(0.973$ to 1.358$)$ & 0.547 \\
Erythrocyte sedimentation rate & $0.971(0.916$ to 1.029$)$ & 0.330 \\
Disease activity score 28 score & $6.510(1.194$ to 35.461$)$ & 0.030 \\
C-reactive protein & $1.136(1.059$ to 1.219$)$ & 0.0004 \\
\hline CI: Confidence interval & & \\
\hline
\end{tabular}


The factors detected in this study to be independently associated with MetS in RA included disease duration, systemic inflammatory marker, mainly CRP, and disease activity. The association between CRP, disease duration, and DAS28 has been previously reported..$^{12,27}$ In our study, we did not find any association between ESR and MetS contrary to other studies. ${ }^{28}$ Rheumatoid factor seropositivity was also not associated with prevalence of MetS in RA. Similar findings were reported by Rostom et al. ${ }^{29}$ and Karvounaris et al. ${ }^{12}$

Our study has some limitations which may be found below;

1. Small sample size compared to other studies,

2. Study's cross-sectional design not allowing to conduct any cause-effect inferences on relationship between RA characteristics and MetS.

3. Risk of selection bias since our center is a tertiary referral center with recruitment of mostly active and severe disease patients,

4. The DAS28 ESR, which was an assessment method used in this study, being only a single point measure of disease activity whereas this will vary over time for RA.

In conclusion, this study showed that MetS is highly prevalent in RA patients than normal population. A higher systemic inflammatory marker, disease duration, and disease activity were independent predictors of MetS in RA. Our findings suggest that physicians should screen RA patients for MetS to check for and reduce risk of cardiovascular diseases.

\section{Declaration of conflicting interests}

The authors declared no conflicts of interest with respect to the authorship and/or publication of this article.

\section{Funding}

The authors received no financial support for the research and/or authorship of this article.

\section{REFERENCES}

1. Masi AT, Medsger TA Jr. Epidemiology of the rheumatic diseases. In: McCarty DJ, editor. Arthritis and Allied Conditions, 11th ed. Philadelphia: Lea \& Febiger; 1989. p. 16-54.

2. Stevens RJ, Douglas KM, Saratzis AN, Kitas GD. Inflammation and atherosclerosis in rheumatoid arthritis. Expert Rev Mol Med 2005;7:1-24.

3. del Rincón ID, Williams K, Stern MP, Freeman GL, Escalante A. High incidence of cardiovascular events in a rheumatoid arthritis cohort not explained by traditional cardiac risk factors. Arthritis Rheum 2001;44:2737-45.

4. McEntegart A, Capell HA, Creran D, Rumley A, Woodward M, Lowe GD. Cardiovascular risk factors, including thrombotic variables, in a population with rheumatoid arthritis. Rheumatology (Oxford) 2001;40:640-4.

5. Sattar N, McCarey DW, Capell H, McInnes IB. Explaining how "high-grade" systemic inflammation accelerates vascular risk in rheumatoid arthritis. Circulation 2003;108:2957-63.

6. Cavagna L, Boffini N, Cagnotto G, Inverardi F, Grosso V, Caporali R. Atherosclerosis and rheumatoid arthritis: more than a simple association. Mediators Inflamm 2012;2012:147354.

7. Kahn R, Buse J, Ferrannini E, Stern M. American Diabetes Association; European Association for the Study of Diabetes. The metabolic syndrome: time for a critical appraisal: joint statement from the American Diabetes Association and the European Association for the Study of Diabetes. Diabetes Care 2005;28:2289-304.

8. Feraaz-Amaro I, Gonzalez-Juanatey C, LopezMejias R, Riancho-Zarrabeitia R, Gonzalez-Gay MA. Metabolic syndrome in rheumatoid arthritis mediators of inflammtion. Mediators of Inflammation Vol. 2013.

9. Misra A, Pandey RM, Devi JR, Sharma R, Vikram NK, Khanna N. High prevalence of diabetes, obesity and dyslipidaemia in urban slum population in northern India. Int J Obes Relat Metab Disord 2001;25:1722-9.

10. Aletaha D, Neogi T, Silman AJ, Funovits J, Felson DT, Bingham CO, et al. 2010 Rheumatoid arthritis classification criteria: an American College of Rheumatology/European League Against Rheumatism collaborative initiative. Arthritis Rheum 2010;62:2569-81.

11. Gremese E, Ferraccioli G. The metabolic syndrome: the crossroads between rheumatoid arthritis and cardiovascular risk. Autoimmun Rev 2011;10:582-9.

12. Karvounaris SA, Sidiropoulos PI, Papadakis JA, Spanakis EK, Bertsias GK, Kritikos HD, et al. Metabolic syndrome is common among middle-toolder aged Mediterranean patients with rheumatoid arthritis and correlates with disease activity: a retrospective, cross-sectional, controlled, study. Ann Rheum Dis 2007;66:28-33.

13. Dodani S, Henkhaus R, Wick J, Vacek J, Gupta K, Dong $\mathrm{L}$, et al. Metabolic syndrome in South Asian immigrants: more than low HDL requiring aggressive management. Lipids Health Dis 2011;10:45. 
14. Zonana-Nacach A, Santana-Sahagún E, JiménezBalderas FJ, Camargo-Coronel A. Prevalence and factors associated with metabolic syndrome in patients with rheumatoid arthritis and systemic lupus erythematosus. J Clin Rheumatol 2008;14:74-7.

15. Dessein PH, Tobias M, Veller MG. Metabolic syndrome and subclinical atherosclerosis in rheumatoid arthritis. J Rheumatol 2006;33:2425-32.

16. Raterman HG, van Eijk IC, Voskuyl AE, Peters MJ, Dijkmans BA, van Halm VP, et al. The metabolic syndrome is amplified in hypothyroid rheumatoid arthritis patients: a cross-sectional study. Ann Rheum Dis 2010;69:39-42.

17. Toms TE, Panoulas VF, John H, Douglas KM, Kitas GD. Methotrexate therapy associates with reduced prevalence of the metabolic syndrome in rheumatoid arthritis patients over the age of 60- more than just an anti-inflammatory effect? A cross sectional study. Arthritis Res Ther 2009;11:110.

18. Elkan AC, Håkansson N, Frostegård J, Cederholm T, Hafström I. Rheumatoid cachexia is associated with dyslipidemia and low levels of atheroprotective natural antibodies against phosphorylcholine but not with dietary fat in patients with rheumatoid arthritis: a cross-sectional study. Arthritis Res Ther 2009;11:37.

19. Chung CP, Oeser A, Solus JF, Avalos I, Gebretsadik $\mathrm{T}$, Shintani A, et al. Prevalence of the metabolic syndrome is increased in rheumatoid arthritis and is associated with coronary atherosclerosis. Atherosclerosis 2008;196:756-63.

20. Aman S, Nilesh B, Kusum S, Indu V. Metabolic syndrome in Indian patients with rheumatoid arthritis and its correlation with disease activity. Arthritis Res Ther 2012;14 (Suppl 1):P66.

21. Cojocaru M, Cojocaru IM, Silosi I, Vrabie CD. Metabolic syndrome in rheumatoid arthritis. Maedica
(Buchar) 2012;7:148-52.

22. Kumar BS, Sivaram Naik G, Katyarmal DT, Prabarath Kumar D, Suresh V, Srinivasa Rao PVLN. Matabolic syndrome. Patients with rheumatoid arthritis:clinical implications. J Clin Sci Res 2013;2:94-100

23. Georgiadis AN, Papavasiliou EC, Lourida ES, Alamanos Y, Kostara C, Tselepis AD, et al. Atherogenic lipid profile is a feature characteristic of patients with early rheumatoid arthritis: effect of early treatment e a prospective, controlled study. Arthritis Res Ther 2006;8:R82.

24. Hassen Zrour S, Hassine Neffeti F, Sakly N, Jguirim $\mathrm{M}$, Korbaa $\mathrm{W}$, Younes $\mathrm{M}$, et al. Lipid profile in Tunisian patients with rheumatoid arthritis. Clin Rheumatol 2011;30:1325-31.

25. Dursunoğlu D, Evrengül H, Polat B, Tanriverdi H, Cobankara V, Kaftan A, et al. Lp(a) lipoprotein and lipids in patients with rheumatoid arthritis: serum levels and relationship to inflammation. Rheumatol Int 2005;25:241-5.

26. Crowson CS, Myasoedova E, Davis JM, Matteson EL, Roger VL, Therneau TM, et al. Increased prevalence of metabolic syndrome associated with rheumatoid arthritis in patients without clinical cardiovascular disease. J Rheumatol 2011;38:29-35.

27. Dao HH, Do QT, Sakamoto J. Increased frequency of metabolic syndrome among Vietnamese women with early rheumatoid arthritis: a cross-sectional study. Arthritis Res Ther 2010;12:218.

28. Alexander CM, Landsman PB, Grundy SM. The influence of age and body mass index on the metabolic syndrome and its components. Diabetes Obes Metab 2008;10:246-50.

29. Rostom S, Mengat M, Lahlou R, Hari A, Bahiri R, Hajjaj-Hassouni N. Metabolic syndrome in rheumatoid arthritis: case control study. BMC Musculoskelet Disord 2013;14:147. 